\title{
Trastornos de la conducta alimentaria y trastornos afectivos: Un estudio comparativo
}

\author{
Rosa Behar A, María Inés Arriagada S, D unny Casanova Za. \\ Eating and affective disorders: \\ A comparative study
}

Background: The relationship between eating disorders and affective disorders still remains unclear. Eating disordered patients may have affective disorders and vice versa, depressed and maniac patients may experience eating problems. Aim: To compare eating symptoms, attitudes and behaviors in patients with affective disorders and normal subjects. Subjects and methods: A structured clinical interview, the Eating Attitudes Test (EAT-40) and the Eating Disorder Inventory (EDI) were administered to 194 patients that fulfilled the DSM-IV diagnostic criteria for eating disorders, to 45 patients with affective disorders and to 82 normal female students. Results: Patients with eating disorders ranked significantly higher on the EAT-40 and on the EDI and its factors ( $p<0.001)$. Patients with affective disorders ranked between eating disordered patients and the students $(p<0.001$ ). Compulsive-purgative type of anorectics and purgative type of bulimics showed the highest scores on these measures ( $\mathrm{p}<0.001$ ). Restrictive type of anorectics scored significantly highest on EDI maturity fears item $(\mathrm{p}<0.001)$. Not significant difference was observed on the EDI ineffectiveness item, between purgative bulimics and depressive patients and between the latter and compulsive-purgative anorexics, on the EDI interpersonal distrust item. Conclusions: Compulsive-purgative type of anorectics and purgative type of bulimics showed the more severe psychological and behavioral disturbances. Restrictive types of anorectics were the most immature. Both purgative bulimics and depressive patients showed feelings of general inadequacy, and both compulsive-purgative anorexics and depressive patients displayed an interpersonal distrust. As a whole, patients with affective disorders did not show the core eating disordered behaviors and attitudes as seen in patients suffering from eating problems (Rev Méd Chile 2005; 133: 1407-14).

(Key Words: Anorexia nervosa; Bulimia; Eating disorders; Mood disorders)

Recibido el 28 de febrero, 2005. Aceptado el 30 de junio, 2005.

Departamento de Psiquiatría, Universidad de Valparaíso. Valparaíso, Chile

aLicenciado en Ciencias y Bioestadístico

Correspondencia a: Dra. Rosa Behar A. Departamento de Psiquiatría, Universidad de Valparaíso. Casilla 92-V, Valparaíso. Fono/Fax: (56) (32) 508550. E mail: rositabehar@vtr.net 
os pacientes con trastornos alimentarios _poseen mayor incidencia de trastornos afectivos que la población general ${ }^{1}$, con una mayor prevalencia de depresión mayor, tanto en la anorexia nerviosa como en la bulimia nerviosa. La frecuente asociación entre ambos cuadros está bien documentada 2,3 . La mayoría de los estudios en este tópico se ha focalizado en la concomitancia entre las patologías alimentarias y la depresión unipolar $^{4-7}$ y el trastorno bipolar, en particular, bipolar II, más habitual en pacientes bulímicas que presentan un tipo crónico y severo de problema alimentario ${ }^{8}$.

La incidencia de una alteración afectiva entre anorécticas oscila entre $52 \%^{9}$ y $98 \% 11$, siendo la depresión el trastorno más común ${ }^{11}$; en las pacientes bulímicas estas cifras fluctúan entre $52 \%$ y $83 \%^{11}$ y para depresión mayor entre 38\% y $63 \%$. La ocurrencia de trastorno afectivo bipolar II entre las anorécticas se ha encontrado desde 9,7\%, en un seguimiento a 10 años plazo ${ }^{9}$, hasta $56 \%$ en pacientes hospitalizadas con desórdenes alimentarios severamente enfermas ${ }^{8}$. Por otra parte, se ha identificado un elevado rango de trastornos depresivos en familiares de pacientes con anorexia nerviosa y bulimia nerviosa, comparados con poblaciones control ${ }^{12,13}$. El trastorno bipolar suele hallarse en familiares de pacientes con anorexia purgativa y bulimia ${ }^{4,6,7}$. Se presume que existe una incidencia cruzada, no apreciándose aumento correspondiente del índice de trastornos alimentarios en los familiares de primer grado con trastornos depresivos, sugiriendo que estos desórdenes no comparten una misma patogénesis y que la transmisión de sendos trastornos no va ligada básicamente ${ }^{12,13}$.

Al presente, la relación entre ambos desórdenes permanece poco clara. Se han barajado varias hipótesis al respecto; algunas afirman que las afecciones alimentarias no son una variante de un trastorno afectivo, ni un equivalente depresivo ${ }^{14}$, sin embargo, estos supuestos han perdido consistencia. Vandereyken ${ }^{15}$ propuso que la anorexia nerviosa y bulimia nerviosa pudieran ser una variante de trastorno afectivo y enfatizó que, a mayor sintomatología de ambas enfermedades, mayor era la patología depresiva. De hecho, muchos pacientes con desórdenes alimentarios informan de «comilonas» como un intento de mejorar su ánimo ${ }^{16}$. También se ha planteado la posibilidad de que factores ambientales y genéticos puedan conducir a un incremento de la vulnerabilidad para ambos desórdenes ${ }^{3,17}$. Se ha argumentado que las bases biológicas que subyacen a estos trastornos y que implican una disfunción adrenérgica y serotoninérgica, pueden ser comunes. La inanición, a su vez, induce síntomas depresivos $^{18}$ que han sugerido similitudes en alteraciones serotoninérgicas entre depresión y trastornos alimentarios. Hudson y Pope ${ }^{19}$ basándose en la respuesta al tratamiento antidepresivo, describen una familia de afecciones bajo el concepto de trastorno del espectro afectivo que incluye, entre otros, la bulimia nerviosa, el trastorno obsesivo-compulsivo, el trastorno de pánico, que son añadidos a la depresión mayor ${ }^{14}$.

Existen escasos trabajos acerca de la incidencia de trastornos alimentarios en trastornos del estado de ánimo. En estudios en los que se ha determinado la relación temporal entre el trastorno alimentario y el trastorno depresivo, sólo en una pequeña minoría de casos se ha apreciado que la alteración del estado de ánimo precede al desorden alimentario 20,21. Aproximadamente la mitad de las personas con anorexia nerviosa afectadas de trastorno depresivo mayor, lo experimentan poco antes de iniciarse la anorexia nerviosa ${ }^{22,23}$. McElroy et $\mathrm{al}^{24}$ en un estudio detectaron que la mayoría de los pacientes bipolares tenía un trastorno comórbido, pero sólo en $5 \%$ éste se trataba de una patología de la conducta alimentaria, entre otros cuadros, asociada con una edad más temprana de inicio de los síntomas afectivos y del trastorno bipolar sindromático. La comorbilidad actual del eje I se asoció con una historia de desarrollo tanto de aceleración cíclica y episodios más severos a través del tiempo.

Los datos epidemiológicos indican que la depresión invernal es más frecuentemente parte de un trastorno bipolar II $^{25-27}$, observándose que la bulimia nerviosa puede mostrar variaciones estacionales con empeoramiento invernal de síntomas bulímicos. En el trastorno afectivo estacional, pueden ocurrir «comilonas», aunque menos frecuentemente episodios purgativos 28,29 , que conducen a una ganancia ponderal significativa en alrededor de $10 \%$ de los pacientes. Otros investigadores, por el contrario, abogan a favor de que se trataría de formas atípicas de trastorno depresivo, con humor disfórico, hiperfagia y exa- 
cerbación de síntomas vegetativos ${ }^{30}$. Fava et $\mathrm{al}^{31}$ evaluaron con el Eating Disorder Inventory $(E D I)^{32}$, posible sintomatología de trastorno alimentario en pacientes con depresión mayor, antes y después del tratamiento (fluoxetina $20 \mathrm{mg} / 24 \mathrm{~h}$ ). Todas las subescalas del cuestionario fueron estadísticamente significativas, demostrando la efectividad de la fluoxetina, sobre todo en las subescalas de bulimia, perfeccionismo, ineficacia y dificultad de las relaciones interpersonales. Los resultados sugieren que los síntomas característicos de desórdenes alimentarios están relacionados con la severidad de la sintomatología depresiva. Cuando aparecía una disminución de los síntomas de patología alimentaria gracias al tratamiento, se observaba una mejoría en el estado anímico de dichos pacientes.

Así como los trastornos de la conducta alimentaria presentan alteraciones del ánimo en su evolución natural, es razonable postular que los trastornos afectivos, a su vez, muestren variaciones del apetito y de los comportamientos alimentarios. El objetivo de esta investigación es efectuar un análisis comparativo entre pacientes de sexo femenino, portadoras de un trastorno de la conducta alimentaria, versus pacientes con trastornos afectivos y sujetos sin ambas patologías, en cuanto a rasgos psicológicos, actitudinales y conductuales descritos como característicos de los trastornos del hábito del comer.

\section{Material y MÉTOdo}

Sujetos. Se dispuso de tres grupos de participantes:

a) Grupo TCA: 194 pacientes de sexo femenino, elegidas al azar entre las que consultaron en el policlínico externo del Hospital del Salvador (Valparaíso), en el Servicio Médico y Dental de Alumnos de la Universidad de Valparaíso y en la práctica privada de los autores y que satisficieron los criterios diagnósticos para trastornos de la conducta alimentaria según el DSM-IV 33 , al menos durante un año previo al estudio (18 anorécticas restrictivas, cuyo rasgo principal era la disminución de la ingestión alimentaria; 67 anorécticas compulsivo-purgativas que recurrían regularmente a «comilonas» o purgas durante sus episodios de anorexia; 39 bulímicas purgativas, las cuales además de presentar «comilonas», se inducían regular- mente el vómito o usaban laxantes o ambas actitudes y 70 pacientes con trastornos de la conducta alimentaria no especificado, que no reunían los requisitos diagnósticos para los síndromes alimentarios completos).

b) Grupo TAF: 45 pacientes de sexo femenino, elegidas al azar entre las que consultaron en la Unidad de Trastornos Afectivos del Hospital del Salvador, que satisficieron los criterios diagnósticos para trastornos afectivos (30 trastorno depresivo mayor, 13 trastorno bipolar I y 2 trastorno bipolar II), al menos durante un año previo al estudio. Se excluyeron aquellas pacientes que llevaban menos de un mes de tratamiento al momento del presente estudio.

c) Grupo E: 82 estudiantes pertenecientes a las carreras de Medicina y de Psicología de la Universidad de Valparaíso, de sexo femenino y que fueron seleccionadas aleatoriamente. Se excluyeron aquellas que habían presentado antecedentes de una patología alimentaria o afectiva 0 la presentaban al momento de esta investigación.

Procedimientos. En la ciudad de Valparaíso, entre junio de 2002 y marzo de 2004, a todas las pacientes con trastorno de la conducta alimentaria, se les realizó una entrevista estructurada confeccionada por los autores y la versión traducida al español y validada del EAT-40 (Test de Actitudes Alimentarias) ${ }^{34}$ y el EDI (Inventario de Desórdenes Alimentarios) ${ }^{32}$ y se aplicó la misma evaluación a los grupos comparativos. Las sujetos en su totalidad respondieron voluntariamente y con consentimiento informado los instrumentos citados. El período de esta investigación se prolongó por el tiempo necesario hasta lograr un tamaño de la muestra adecuado para la exploración. Estos se aplicaron, por una sola vez en cada sujeto, para ser contestados en un lapso de aproximadamente 45 minutos en forma individual y durante una única jornada. La coautora entrevistadora (MA) recibió entrenamiento previo de parte de la autora principal (RB) mediante cursos y seminarios, como parte de su formación en la especialidad de psiquiatría.

1. La entrevista estructurada incluyó los datos de edad, peso actual, talla, peso ideal y preguntas basadas en los criterios diagnósticos del DSMIV $^{33}$ para trastornos de la conducta alimentaria, lo que permitió pesquisar o confirmar la 
presencia de esta patología en los grupos comparativos.

2. El Test de Actitudes Alimentarias (Eating Attitudes Test), EAT-4034, posee 40 preguntas distribuidas en 3 factores que miden 1) dieta: evitación de alimentos que engordan; 2) control oral: control en el comer y presión percibida por parte de los demás para aumentar de peso y; 3) bulimia y preocupación por el alimento: pensamientos relacionados con los alimentos y conductas bulímicas. Cada respuesta tiene un valor máximo de 3 puntos. Un puntaje igual o superior a 30 en el EAT-40 es indicativo de un trastorno del hábito del comer, anoréctico o bulímico, ya sea clínicamente existente 0 encubierto, que pudiera desencadenarse bajo ciertas circunstancias propicias.

3. El EDI ${ }^{32}$ consiste en 64 proposiciones distribuidas en 8 ítemes que evalúan: 1) Motivación por la delgadez (MD): la excesiva preocupación por el peso y la dieta; 2) Conciencia interoceptiva (CI): la falta de capacidad para identificar las propias emociones y sensaciones; 3) Bulimia (B): la presencia de «comilonas» y purgas; 4) Insatisfacción corporal (IC): la distorsión de la imagen corporal; 5) Sensación de inutilidad (I): sentimientos de minusvalía e inseguridad; 6) Temor a la madurez (TM): regresión a los años preadolescentes frente a las demandas de la adultez; 7) Perfeccionismo (P): las excesivas expectativas personales hacia logros superiores y; 8) Desconfianza interpersonal (DI): una resistencia a establecer relaciones cercanas. El EDI tiene un máximo de 3 puntos para cada respuesta, no se consideró en su ponderación global un rango de corte y en consecuencia se analizó cada ítem en forma independiente.

Estadística. La descripción de cada uno de los tres grupos se efectuó para variables cuantitativas (edad, talla, peso actual, IMC [Índice de Masa Corporal], peso ideal, EAT-40, EDI y sus 8 ítemes), mediante promedios, desviaciones estándar y correlaciones de Pearson. La comparación entre grupos para cada variable cuantitativa se realizó mediante análisis de varianza (ANOVA) y test Kruskal-Wallis.

\section{Resultados}

Ciento diecinueve $(61,3 \%)$ de las integrantes del grupo TCA poseían una educación universitaria, 58 de ellas $(29,9 \%)$ un nivel de enseñanza media, $16(8,2 \%)$ un grado de educación técnica y 1 $(0,6 \%)$ sólo había alcanzado la enseñanza básica. La escolaridad del grupo TAF se distribuyó en 7 con educación universitaria (15,6\%), 23 con enseñanza media $(51,1 \%), 8$ con nivel técnico $(17,8 \%)$ y 7 con enseñanza básica (15,6\%). Respecto al estado civil, todas las integrantes del grupo TCA eran solteras, mientras que del grupo TAF sólo 15 eran solteras (33,3\%), 20 eran casadas $(44,5 \%)$ y 10 separadas $(22,2 \%)$.

Considerando los 7 grupos iniciales, el grupo de sanas, sin patología alimentaria (SPA) presentó los menores valores tanto para el indicador EAT40 como para EDI y sus ítemes componentes, siendo por lo general las anorécticas compulsivas (ACP) y las bulímicas purgativas (BUP) las que exhibieron los mayores valores de estos indicadores. Estas diferencias fueron siempre significativas ( $p<0,001$ ) (Tabla 1). En cuanto a edad, pesos e IMC, los mayores valores estuvieron en las pacientes con trastornos afectivos, ya sea depresión mayor (DEM) o bipolares (BIP) y los menores en las pacientes anorécticas compulsivas y restrictivas, con diferencias también significativas ( $p$ $<0,001)$. Las únicas diferencias que no fueron significativas fueron entre tallas (Tabla 1).

Resumiendo en un solo grupo las mujeres con patología alimentaria (ACP+ARE+TAN+BUP) y en otro grupo a las con trastornos afectivos (DEM+BIP), se pudo observar que para todas las variables, la diferencia entre los 3 grupos se mantuvo significativa. Los mayores valores de los indicadores EAT-40, EDI y sus ítemes componentes, se encontraron en el grupo de mujeres con patología alimentaria (CPA) y los menores en el sin patología alimentaria (SPA), quedando en el centro los con trastornos afectivos (TAF) (Tabla 2). La edad, PA, PI e IMC, fuemon mayores en el grupo con trastornos afectivos (TAF) y similar en los otros dos grupos (Tabla 2).

\section{DisCUSIÓN}

Nuestros hallazgos confirman la patología alimentaria clínicamente diagnosticada en el grupo de las 
Tabla 1. Promedio y desviación estándar para variables cuantitativas, en cada grupo

\begin{tabular}{|c|c|c|c|c|c|c|c|c|c|c|c|c|c|c|}
\hline & \multicolumn{2}{|c|}{$\begin{array}{c}\mathrm{ACP} \\
(\mathrm{n}=67)\end{array}$} & \multicolumn{2}{|c|}{$\begin{array}{c}\text { ARE } \\
(\mathrm{n}=18)\end{array}$} & \multicolumn{2}{|c|}{$\begin{array}{c}\text { TAN } \\
(\mathrm{n}=70)\end{array}$} & \multicolumn{2}{|c|}{$\begin{array}{c}\text { BUP } \\
(\mathrm{n}=39)\end{array}$} & \multicolumn{2}{|c|}{$\begin{array}{c}\text { SPA } \\
(n=82)\end{array}$} & \multicolumn{2}{|c|}{$\begin{array}{c}\text { DEM } \\
(\mathrm{n}=30)\end{array}$} & \multicolumn{2}{|c|}{$\begin{array}{c}\text { BIP } \\
(n=15)\end{array}$} \\
\hline & $\overline{\mathrm{x}}$ & $\mathrm{DE}$ & $\overline{\mathrm{x}}$ & $\mathrm{DE}$ & $\overline{\mathrm{x}}$ & $\mathrm{DE}$ & $\overline{\mathrm{x}}$ & $\mathrm{DE}$ & $\overline{\mathrm{x}}$ & $\mathrm{DE}$ & $\overline{\mathrm{x}}$ & $\mathrm{DE}$ & $\overline{\mathrm{x}}$ & $\mathrm{DE}$ \\
\hline Edad (años) & 20,1 & 3,9 & 21,5 & 9,1 & 21,9 & 5,5 & 22,9 & 6,4 & 22,2 & 2,3 & 38,4 & 11,1 & 39,6 & 7,6 \\
\hline EAT-40 & 59,6 & 20,0 & 43,7 & 19,7 & 44,4 & 20,8 & 51,7 & 17,6 & 10,9 & 6,3 & 28,1 & 15,2 & 23,5 & 11,1 \\
\hline EDI & 91,5 & 31,3 & 64,4 & 23,0 & 83,0 & 31,3 & 96,7 & 25,5 & 24,2 & 17,8 & 68,6 & 35,2 & 49,1 & 24,5 \\
\hline $\mathrm{MD}$ & 16,0 & 5,8 & 10,3 & 7,5 & 13,9 & 5,9 & 15,4 & 5,0 & 2,5 & 3,9 & 8,4 & 6,4 & 6,0 & 4,4 \\
\hline $\mathrm{CI}$ & 13,7 & 6,3 & 9,1 & 7,4 & 13,1 & 7,6 & 14,8 & 6,1 & 1,3 & 2,6 & 12,6 & 8,8 & 6,0 & 5,1 \\
\hline B & 9,2 & 5,8 & 3,9 & 3,8 & 8,2 & 5,4 & 11,4 & 6,0 & 2,6 & 2,0 & 4,8 & 4,1 & 3,5 & 3,2 \\
\hline IC & 15,9 & 8,1 & 10,8 & 7,3 & 14,9 & 7,9 & 17,9 & 7,3 & 4,8 & 6,2 & 11,1 & 8,0 & 10,7 & 8,7 \\
\hline I & 9,4 & 7,4 & 5,1 & 5,2 & 8,1 & 7,1 & 10,9 & 7,8 & 0,9 & 2,1 & 10,2 & 7,2 & 7,2 & 6,8 \\
\hline TM & 10,6 & 5,6 & 12,0 & 6,8 & 11,0 & 5,9 & 11,3 & 6,1 & 4,2 & 4,3 & 8,0 & 5,9 & 6,4 & 4,3 \\
\hline $\mathrm{P}$ & 9,7 & 4,3 & 8,2 & 3,4 & 9,7 & 4,8 & 9,4 & 3,9 & 6,5 & 4,3 & 7,3 & 4,4 & 6,9 & 4,3 \\
\hline DI & 6,6 & 5,6 & 5,1 & 4,5 & 4,3 & 3,9 & 5,7 & 4,5 & 1,5 & 2,2 & 6,1 & 5,7 & 2,8 & 3,4 \\
\hline $\mathrm{PA}$ & 51,2 & 7,5 & 47,6 & 8,2 & 59,6 & 13,0 & 59,9 & 11,2 & 55,7 & 6,1 & 67,1 & 11,6 & 68,7 & 12,8 \\
\hline Talla $(\mathrm{cm})$ & 160,4 & 6,6 & 160,2 & 6,7 & 160,4 & 5,4 & 160,5 & 5,5 & 161,2 & 5,5 & 158,5 & 5,9 & 158,3 & 6,3 \\
\hline PI & 48,7 & 5,6 & 49,1 & 6,2 & 52,8 & 6,3 & 52,0 & 5,4 & 53,5 & 4,8 & 56,6 & 6,3 & 57,5 & 6,7 \\
\hline IMC $\left(\mathrm{kg} / \mathrm{mt}^{2}\right)$ & 19,9 & 2,4 & 18,5 & 3,0 & 23,1 & 4,6 & 23,2 & 4,1 & 21,4 & 1,8 & 26,7 & 4,2 & 27,3 & 4,3 \\
\hline & & & & & & & & & & & & & & \\
\hline & & & & & & & $\mathrm{ARE}=$ & & & & stricti & & & \\
\hline & & & & ia no & & & $\mathrm{BUP}=$ & $\mathrm{Bul}$ & & sa p & gativa & & & \\
\hline $\mathrm{PA}=$ Sin trasto & de la & nduc & a alime & taria & & & $\mathrm{DEM}=$ & Dep & esión n & & & & & \\
\hline
\end{tabular}

Tabla 2. Promedio y desviación estándar de variables cuantitativas en cada grupo resumido

\begin{tabular}{|lrrrrrrrr|}
\hline & \multicolumn{2}{c}{ CPA $(\mathrm{n}=194)$} & \multicolumn{2}{c}{ SPA $(\mathrm{n}=82)$} & \multicolumn{2}{c|}{ TAF $(\mathrm{n}=45)$} & \multicolumn{2}{c|}{ Total $(\mathrm{n}=321)$} \\
& $\overline{\mathrm{x}}$ & $\mathrm{DE}$ & $\overline{\mathrm{x}}$ & $\mathrm{DE}$ & $\overline{\mathrm{x}}$ & $\mathrm{DE}$ & $\overline{\mathrm{x}}$ & $\mathrm{DE}$ \\
\hline Edad (años) & 21,4 & 5,7 & 22,2 & 2,3 & 38,8 & 9,9 & 24,1 & 8,4 \\
EAT-40 & 51,0 & 20,8 & 10,9 & 6,3 & 26,6 & 14,0 & 37,4 & 24,6 \\
EDI & 87,0 & 30,7 & 24,2 & 17,8 & 62,1 & 33,1 & 67,4 & 38,9 \\
MD & 14,6 & 6,0 & 2,5 & 3,9 & 7,6 & 5,9 & 10,6 & 7,6 \\
CI & 13,3 & 7,0 & 1,3 & 2,6 & 10,4 & 8,2 & 9,8 & 8,1 \\
B & 8,8 & 5,8 & 2,6 & 2,0 & 4,4 & 3,8 & 6,6 & 5,6 \\
IC & 15,5 & 7,9 & 4,8 & 6,2 & 11,0 & 8,1 & 12,1 & 8,8 \\
I & 8,8 & 7,0 & 3,9 & 2,1 & 9,2 & 7,1 & 6,9 & 7,3 \\
TM & 11,0 & 5,9 & 4,2 & 4,9 & 7,5 & 5,3 & 8,8 & 6,2 \\
P & 9,5 & 4,3 & 6,5 & 4,3 & 7,2 & 4,3 & 8,4 & 4,5 \\
DI & 5,4 & 4,8 & 1,5 & 2,2 & 5,0 & 5,2 & 4,4 & 4,7 \\
PA & 55,7 & 11,5 & 55,7 & 6,1 & 67,7 & 11,9 & 57,4 & 11,2 \\
Talla (cm) & 160,4 & 6,0 & 161,2 & 5,5 & 158,4 & 5,9 & 160,3 & 5,9 \\
PI & 50,8 & 6,1 & 53,5 & 4,8 & 56,9 & 6,4 & 52,4 & 6,2 \\
IMC (kg/mt $\left.{ }^{2}\right)$ & 21,5 & 4,1 & 21,4 & 1,8 & 26,9 & 4,2 & 22,3 & 4,1 \\
\hline
\end{tabular}

$\overline{\mathrm{X}}=$ Promedio $\mathrm{DE}=$ Desviación estándar

$\mathrm{CPA}=$ Con trastorno de la conducta alimentaria $\quad \mathrm{SPA}=$ Sin trastorno de la conducta alimentaria

$\mathrm{TAF}=$ Con trastorno afectivo 
pacientes, en contraste con las pacientes depresivas, bipolares I, II y estudiantes no portadoras de una afección alimentaria ni de un trastorno del ánimo. El menor IMC lo presentaron las anorécticas restrictivas y el mayor las pacientes bipolares. Sin embargo, el peso ideal más bajo fue concebido por las pacientes anorécticas compulsivopurgativas.

Dentro de las pacientes con trastornos alimentarios, las anorécticas compulsivo-purgativas y las bulímicas purgativas, fueron las más patológicas. Las más motivadas para alcanzar la delgadez, las más perfeccionistas (en conjunto con las pacientes portadoras de un trastorno de la conducta alimentaria no especificado) y con mayor desconfianza interpersonal fueron las anorécticas compulsivopurgativas. En cambio, las que tuvieron más dificultades para discriminar sus emociones y sentimientos y las que presentaron más conductas de «comilonas»y purgas, más insatisfacción corporal y sensación de ineficacia personal, fueron las bulímicas purgativas. Sin embargo, de todos los subgrupos con desórdenes alimentarios, las anorécticas restrictivas mostraron más temores a la madurez que cualquiera de los otros subtipos. Al respecto, Crisp ${ }^{35}$ ha sugerido que una de las manifestaciones sintomatológicas centrales de la anorexia nerviosa es el retroceso a la seguridad de los años preadolescentes debido a las abrumadoras exigencias de la adultez, a través de la evitación de la ingesta de carbohidratos.

Siendo la motivación por la delgadez el índice más sensible y específico para predecir un trastorno de la conducta alimentaria ${ }^{36}$, tanto las pacientes con trastornos del ánimo como las estudiantes catalogadas como sanas, al presentar una baja motivación para alcanzar una figura corporal delgada, están protegidas para el desarrollo de una afección alimentaria.

Respecto a las pacientes con trastornos afectivos, en los puntajes globales tanto del EAT-40 como del EDI con sus ítemes, las pacientes depresivas se mostraron más sanas en todos los indicadores de conducta patológica alimentaria, en comparación con las pacientes con trastornos del hábito del comer. No obstante, al comparar las pacientes depresivas con las bipolares, las primeras presentan puntajes más elevados en todos los factores del EDI, apareciendo las bipolares, más sanas. La sensación de ineficacia personal en las pacientes depresivas surge como el aspecto más relevante, incluso no siendo significativa la diferencia con el puntaje alcanzado por las pacientes bulímicas purgativas. Es decir, tanto las pacientes bulímicas como las pacientes con trastorno depresivo mayor, poseen de manera similar, sentimientos de inadecuación general, inseguridad, inutilidad y la sensación de no estar en control de sus propias vidas. Este rasgo ha sido descrito como la alteración fundamental en la anorexia nerviosa ${ }^{37}$ y forma parte de los criterios diagnósticos para el trastorno depresivo mayor, tanto en el $\mathrm{DSM}^{-I^{33}}$ como en la CIE-10 ${ }^{38}$. Se ha sugerido asimismo que el concepto de ineficacia también incluye un componente de autoevaluación negativa (auto-concepto) ${ }^{39}$ en las pacientes anorécticas. Conjuntamente, como rasgo común, tanto las anorécticas compulsivo-purgativas como las pacientes depresivas tuvieron una sensación de alienación y rechazo general a establecer relaciones cercanas, que ha sido identificado como un rasgo importante en el desarrollo y mantención de la anorexia nerviosa ${ }^{37,40} \mathrm{y}$, a su vez, figura dentro de los requerimientos diagnósticos para el trastorno depresivo mayor ${ }^{38}$. Surge la interrogante de si estos atributos clínicos son o no específicos de un trastorno afectivo o de un trastorno del comportamiento alimentario, o más bien podrían considerarse como dimensiones orientadas a la estructura y funcionamiento de la personalidad en ambos tipos de pacientes.

La implicancia más relevante de este trabajo es el análisis de la presencia de manifestaciones psicológicas, comportamentales y actitudinales que suelen observarse en pacientes con patologías alimentarias, en pacientes con trastornos afectivos, las cuales en esta exploración aparecen libres de aquellas alteraciones intrínsecas a la anorexia nerviosa, a la bulimia nerviosa o a los trastornos de la conducta alimentaria no especificados, como comorbilidad acompañante. Sólo la sensación de ineficacia personal y la desconfianza interpersonal son expresiones clínicas compartidas por ambos desórdenes, específicamente entre las pacientes depresivas y las bulímicas purgativas y entre las primeras y las anorécticas compulsivopurgativas. Como limitante cabe mencionar la escasez de literatura tanto nacional como extranjera, concerniente a la presencia o detección de problemas alimentarios en pacientes afectivos y 
que reduce las posibilidades de impresiones clínicas acerca de ellos. Además, no se consideró una correlación con el tiempo ni modalidad de manejo terapéutico, ni tampoco la comorbilidad física 0 psicopatológica coexistente en las pacientes con desórdenes del ánimo y que pudiesen influir en los hallazgos.

Al no existir las anomalías previamente analizadas en este estudio, entre las pacientes depresivas y bipolares, a pesar que el promedio del rango de sus puntuaciones fue mayor que el grupo de

\section{REFERENCIAS}

1. Herzog DB, Keluer MB, Sacks NR, Yeh CJ, Lavori PW. Psychiatric comorbidity in treatment-seeking and bulimics. J Am Acad Child Adolesc Psychiatr 1992; 31: 810-8.

2. Cantwell DP, Sturzenberger S, Burroughs J. Anorexia nervosa: An affective disorder? Arch Gen Psychiatry 1977; 34: 1087-93.

3. Swift WJ, Andrews D, Barkiage NE. The relationship between affective disorder and eating disorders: A review of the literature. Am J Psychiatry 1986; 143: 290-9.

4. Greenberg BR, Harvey PD. Affective lability versus depression as determinants of binge eating. Addict Behav 1987; 12: 357-61.

5. Hudson JI, Pope HG. Affective spectrum disorder: Does antidepressant response identify a family of disorders with a common pathophysiology? Am J Psychiatry 1991; 148: 548.

6. Kassett JA, Gerson eS, Maxwell ME, Guroff J, KazuBa D, SMith A et al. Psychiatric disorders in the first-degree relatives of probands with bulimia nervosa. Am J Psychiatry 1989; 146: 1468-71.

7. WINOKUR G. Is there a common genetic factor in bipolar and unipolar affective disorder? Compr Psychiatry 1980; 21: 460-8.

8. Simpson SG, Al-Muffi R, Anderson AE, DeE Paulo JR. Bipolar II affective disorders in eating disordered inpatients. J Nerv Ment Dis 1992; 180: 719.

9. Halmi KA, Eckert E, Marchi P, Sampugnaro V, Apple R, CoHen J. Comorbidity of psychiatric diagnoses in anorexia nervosa. Arch Gen Psychiatry 1991; 48: $712-8$. estudiantes catalogadas como sanas, tal vez sea conveniente evaluar reflexivamente la elevada incidencia descrita en las publicaciones de trastomos afectivos en el contexto clínico de una afección alimentaria. Estimamos que deben desarrollarse más investigaciones en el futuro acerca de la detección de desórdenes de la alimentación en pacientes que sufren trastornos del ánimo y viceversa, su pronóstico y los alcances respecto a las respuestas terapéuticas de la comorbilidad del eje I, en ambas entidades sintomatológicas.

10. Rastam M, Giwberg C, Giшberg C. Anorexia nervosa 6 years after onset: II. Comorbid psychiatric problems. Compr Psychiatry 1995; 36: 70.

11. Herzog D, Nussbaum K, Marmor A. Comorbidity and outcome in eating disorders. Psychiatr Clin North Am 1996; 19: 843-59.

12. Luenfeld L, Kaye WH, Greeno C, Merikangas K, Plotnicov K, Powice C et al A controlled family study of anorexia nervosa and bulimia nervosa: Psychiatric disorders in first-degree relatives and effects of proband comorbidity. Arch Gen Psychiatry 1998; 55: 603-10.

13. Strober M, Lampert C, Morrell W, Burroughs J, JacoBs C. A controlled family study in anorexia nervosa: Evidence of familial aggregation and lack of shared transmision with affective disorders. Int J Eat Disord 1990; 9: 239-53.

14. Снinchiша A. Anorexia y bulimia nerviosas. Madrid: Ergon, 1994.

15. VANDEREYCKEN W. Are anorexia nervosa and bulimia variants of affective disorders? Acta Psychiatrica Belga 1987; 87: 267-80.

16. Kaye WH, Gwirtsman HE, George DT, Weiss SR, JIMERSON DC. Relationships of mood alterations to binging behavior in bulimia. Br J Psychiatry 1986; 149: 479-85.

17. Katz JL, Kuperberg A, Poшack CP, Walsh BT, Zumoff $\mathrm{B}, \mathrm{WEINER} H$. Is there a relationship between eating disorder and affective disorder? New evidence from sleep recordings. Am J Psychiatry 1984; 141: 753-9.

18. Jimerson DC, LeSEM MD, Kaye WH, HegG AP, BREWERTON TD. Eating disorders and depression: Is there a serotonin connection? Biol Psychiatry 1990; 28: 443-54. 
19. Hudson JI, Pope HG. Fibromyalgia and psychopathology: Is fibromyalgia a form of affective spectrum disorder? J Rheumatol 1989; 16: 15-22.

20. COOPER PJ, FAIRBURN CG. The depression symptoms of bulimia nervosa. Br J Psychiatry 1986; 148: 268-74.

21. Laessle RG, KirTl S, Fichter MM, Wittchen HU, PIRKE KM. Major affective disorder in anorexia nervosa and bulimia: A descriptive diagnostic study. Psychiatry 1987; 151: 785-9.

22. Toro J, Vilardell E. Anorexia nerviosa. Barcelona, Martínez Roca, 1987.

23. Cervera S, Quintanila B. Anorexia nerviosa. Manifestaciones psicopatológicas fundamentales. EUNSA. Pamplona: Ediciones Universidad de Navarra S.A., 1995.

24. McElroy S, Altshuler L, Suppes T, Keck P, Frye M, Denicoff K ET AL. Axis I Psychiatric comorbidity and its relationship to historical illness variables in 288 patients with bipolar disorder. Am J Psychiatry 2001; 158: 420-6.

25. Levitan RD, Kaplan AS, LevitT AJ, Joffe RT. Seasonal fluctuations in mood and eating behavior in bulimia nervosa. IntJ Eat Disord 1994; 16: 255-99.

26. GRUBer NP, Dilsaver SC. Bulimia and anorexia nervosa in winter depression: Lifetime rates in a clinical sample. J Psychiatr Neurosc 1996; 21: 9-12.

27. Mury M, Verdoux H, Bourgeois M. Comorbidity of bipolar and eating disorders. Epidemiologic and therapeutic aspects. Encephale 1995; 21: 545-53.

28. Berman K, Lam RW, Goldner EM. Eating attitudes in seasonal affective disorder and bulimia nervosa. J Affect Disord 1993; 29: 219-25.

29. Krauchi K, Reich S, Wirz-Justice A. Eating style in seasonal affective disorder: Who will gain weight in winter? Compr Psychiatry 1997; 38: 80-7.

30. Lebowitz MR, Quitkin F, Steward JW. Phenelzine and imipramine in atypical depression. Psychopharmacological Bull 1981; 17: 159-61.

31. Fava M, Abraham M, Clancy-Colecchi K, Pava JA, Matthews J, Rosenbaum JF. Eating disorder symptomatology in major depression. J Nerv Ment Dis 1997; 185: 140-4.

32. Garner D, Olmstead M, Polivy J. Development and validation of a multidimensional eating disorder inventory for anorexia nervosa and bulimia. Int J Eat Disord 1983; 2: 15-34.

33. American Psychiatric Association. Diagnostic and statistical manual of mental disorders. $4^{\text {th }}$ ed. Washington (DC): American Psychiatric Association; 1994.

34. Garner D, Olmstead M, Bohr I, Gareinkel P. The eating attitudes test: Psychometric features and clinical comelates. Psychological Medicine 1982; 12: 871-8.

35. CRISP AH. Anorexia nervosa. Let me be. London: Academic Press, 1980.

36. BeHAR R. Trastornos del hábito del comer en adolescentes: Aspectos clínicos y epidemiológicos. Rev Méd Chile 1998; 126: 1085-92.

37. BEHAR R. Trastornos de la alimentación y personalidad anormal. En: Riquelme R, Oksenberg A, editores. Trastornos de personalidad. Hacia una mirada integral. Santiago de Chile: Sociedad Chilena de Salud Mental, 2003; 331-48.

38. Wordd Health Organization. The ICD-10 classification of mental and behavioral disorders: Clinical descriptions and diagnostic guidelines. Geneve: WHO, 1992.

39. BRuCH H. Eating disorders. Obesity, anorexia and the person within. New York: Basic Books, 1973.

40. BeHAR R. Trastornos de la conducta alimentaria: Clínica y epidemiología. En: Behar R, Figueroa G, editores. Anorexia nerviosa y bulimia. Clínica y terapéutica. Santiago de Chile: Mediterráneo, 2004.

Agradecimientos

Los autores agradecen a todas aquellas personas que prestaron su valiosa colaboración en esta investigación, sin la cual no habría podido realizarse. 